\title{
Isovector properties of the nuclear equation of state from the quark-meson coupling model
}

\author{
Ellen McRae*, Cedric Simenel, E. C. Simpson, \\ The Australian National University \\ E-mail: ellen.mcraedanu.edu.au
}

\section{Anthony W. Thomas}

The University of Adelaide

\begin{abstract}
The nucleon-nucleon interaction is an important requirement for investigations of nuclear structure and reactions, as well as for astrophysical models such as r-process nucleosynthesis and neutron stars. The traditional approach to low-energy nuclear physics is to treat nucleons as immutable objects interacting via phenomenological forces. The use of phenomenological interactions, rather than one derived from a microscopic theory, raises questions as to the reliability of predictions for exotic regions of the nuclear chart. The quark-meson coupling (QMC) model uses a relativistic mean-field approach to provide a microscopically derived nucleon-nucleon interaction, which takes into account the quark structure of the nucleon.

The Skyrme energy density functional is a popular phenomenological tool in studies of nuclear structure and reactions. In this work, the QMC density functional was used to produce a set of Skyrme parameterisations, in the hopes that they will give more reliable predictions for exotic nuclei. In conjunction with Hartree-Fock-Bogoliubov (HFB) calculations, the Skyrme-QMC (SQMC) parameterisations have been used to model the ground-state properties of individual nuclei. From this, one can investigate the importance of the isovector terms of the nucleon-nucleon interaction, which are particularly significant for exotic, neutron-rich regions of the nuclear chart. One of the notable successes of the QMC model is its derivation of nuclear spin-orbit coupling. The isovector dependence of the spin-orbit equation of state is remarkably similar to that of the modern UNEDF1 phenomenological density functional. HFB calculations along the Sn isotopic chain reveal that the isovector properties of the spin-orbit term impact binding energies to a level that will be significant for astrophysical r-process modelling.
\end{abstract}

The 26th International Nuclear Physics Conference

11-16 September, 2016

Adelaide, Australia

*Speaker. 


\section{Introduction}

One of the main directions of nuclear physics is to extend our understanding of exotic systems, away from the line of stability and towards superheavy elements, the astrophysical r-process path or the neutron dripline. Little to no experimental data exists for these systems. Theoretically, one of the most successful tools currently used is the Skyrme energy density functional. It gives the energy density at each point in the nuclear system, as a function of the local nucleon densities. The functional is derived from the Skyrme interaction [1], which is the most general two-body, zero-range nucleon-nucleon interaction up to second-order in derivatives. The downside of the Skyrme density functional is that it is phenomenological. Its set of 10-17 parameters are typically determined by a fit to experimental masses and radii of nuclides near stability, calling into question the predictions for very exotic systems. A possible solution to this is to obtain the interaction between nucleons from the more fundamental, high-energy degrees of freedom of the system.

The quark-meson coupling (QMC) model is a relativistic mean-field approach to the quantum many-body problem $[2,3]$. Three quarks are confined to a nucleon bag, and the quarks of different bags interact by exchanging $\sigma, \omega$ and $\rho$ mesons. While the nucleons are assumed to move slowly, the quarks are treated relativistically and the model naturally includes certain relativistic corrections. Most notably, the model derives nuclear spin-orbit coupling, a relativistic effect essential for shell structure of nuclei and dissipation in collisions.

\section{Spin-orbit energy density functional}

The spin-orbit energy density functional from the QMC model is given by [3]

$$
\begin{aligned}
\mathscr{H}_{\mathrm{SO}}^{\mathrm{QMC}}= & \frac{-1}{4 M_{N}^{2}}\left[\left(G_{\sigma}+G_{\omega}\left(2 \mu_{s}-1\right)\right) \rho \nabla \cdot \boldsymbol{J}\right. \\
& \left.+\left(\frac{G_{\sigma}}{2}+\frac{G_{\omega}}{2}\left(2 \mu_{s}-1\right)+\frac{3 G_{\rho}}{8}\left(2 \mu_{v}-1\right)\right) \sum_{q=n, p} \rho_{q} \nabla \cdot \boldsymbol{J}_{q}\right],
\end{aligned}
$$

where $\rho_{n}\left(\rho_{p}\right)$ is the neutron (proton) particle density and $\rho$ is the total particle density, $\rho=\rho_{n}+\rho_{p}$; similarly for the spin-orbit density, $\boldsymbol{J}$. The coefficients are formed from the meson coupling constants $\left(G_{\sigma}, G_{\omega}, G_{\rho}\right)$, the isoscalar and isovector nucleon magnetic moments ( $\mu_{s}$ and $\mu_{v}$, respectively), and the free nucleon mass $\left(M_{N}\right)$. Though not strictly necessary, the $\omega$ and $\rho$ meson fields can be identified with real particles and their experimental masses used. This leaves only four free parameters in the QMC model, a coupling constant for each meson field and the mass of the $\sigma$ meson, all of which are fixed by the central terms of the functional. Unlike non-relativistic approaches such as Skyrme, the QMC spin-orbit functional has no extra spin-orbit parameters to adjust. The small number of free parameters in the QMC model is expected to make it more predictive than a standard Skyrme functional, particularly for the spin-orbit term.

The most general form of the Skyrme density functional [4],

$$
\mathscr{H}_{\mathrm{SO}}^{\mathrm{Skyrme}}=-\frac{1}{2}\left[W_{0} \rho \nabla \cdot \boldsymbol{J}+W_{0}^{\prime} \sum_{q=n, p} \rho_{q} \nabla \cdot \boldsymbol{J}_{q}\right],
$$




\begin{tabular}{|l|l|}
\hline$W_{0}^{\prime} / W_{0}$ & Model \\
\hline 1 & Standard Skyrme \\
1.86 & Modern Skyrme: UNEDF1 [5] \\
1.78 & QMC \\
& \\
0.1 & Standard relativistic mean-field (RMF) [6] \\
0.2 & QMC without exchange (Fock) terms and $\mu_{s}=\mu_{v}=1$ \\
\hline
\end{tabular}

Table 1: Ratio of the spin-orbit coupling constants illustrating the isovector dependence of spin-orbit energy density functionals.

takes the same form of the nucleon densities but now with free coefficients $W_{0}$ and $W_{0}^{\prime}$. As the Skyrme interaction is non-relativistic, obeying Galilean rather than Lorentz invariance, the coefficients of the spin-orbit term are completely unknown and must be adjusted to experiment.

The isovector dependence of the spin-orbit functionals quantifies how they are affected by differences between proton and neutron densities, which become increasingly important for studying very neutron rich nuclei. It is controlled by the second term of equations 2.1 and 2.2, as the proton and neutron densities appear individually. Therefore, the ratio of the two coefficients can be used to compare the isovector properties different approaches, as done in Table 1.

Traditionally, Skyrme functionals only had one spin-orbit parameter, $W_{0}$. The modern Universal Nuclear Energy Density Functional (UNEDF1) [5] released this constraint, and from its state-of-the-art fitting procedure, had actually derived a much stronger isovector dependence. The QMC model, with its more fundamental, relativistic basis gives a very similar value.

On the other hand, standard RMF, a relativistic mean-field model of inert nucleons, gives a substantially weaker isovector dependence. By keeping only the direct (Hartree) terms of the QMC spin-orbit functional and setting the nucleon magnetic moments to their Dirac $\left(\mu_{s}=\mu_{v}=1\right)$ rather than experimental $\left(\mu_{s}=0.88, \mu_{v}=4.7\right)$ values, one would obtain a similarly weak isovector dependence. This implies that the weak isovector dependence of RMF is primarily due to these two approximations, neglect of exchange terms and use of Dirac magnetic moments. These are approximations which are not required in the QMC model.

There is a remarkable similarity between the spin-orbit functionals of UNEDF1 and the QMC model. However, the significance of this fact is dependent upon whether the isovector dependence of the spin-orbit term alone has any impact on Hartree-Fock calculations of nuclei. The following section investigates the use of a Skyrme parameterisation from the QMC model and the importance of the isovector properties of the spin-orbit term.

\section{Hartree-Fock calculations}

There exist many codes based on Hartree-Fock approximations which can be used to compute properties of nuclear structure and reactions. The majority of them are based on Skyrme functionals. The QMC functional has similarities to the Skyrme functional but is not identical. We have constructed a Skyrme functional which is as close as possible to that of the QMC model, called Skyrme-QMC (SQMC). A similar SQMC was obtained in Ref. [7], however with $W_{0}=W_{0}^{\prime}$. Here, 
our SQMC has $W_{0}^{\prime} / W_{0}=1.78$ as in the original QMC model. For the central part of the functionals there is a difference in the density dependence and they are matched around the saturation point of nuclear matter. But for the spin-orbit term, SQMC and the QMC model are identical, making SQMC a convenient tool to test the isovector dependence of the original QMC functional.

The axial Hartree-Fock-Bogoluibov code, HFBTHO [8], was used to calculate the groundstate binding energies of tin $(Z=50)$ nuclei. Relative to experimental data [9], as seen in Figure 1, the SQMC functional performs at a level comparable to UNEDF1, especially away from stability. This is particularly remarkable when one recalls that the stable tin masses were included in the fit of UNEDF1, while no experimental masses were used to derive the SQMC functional.

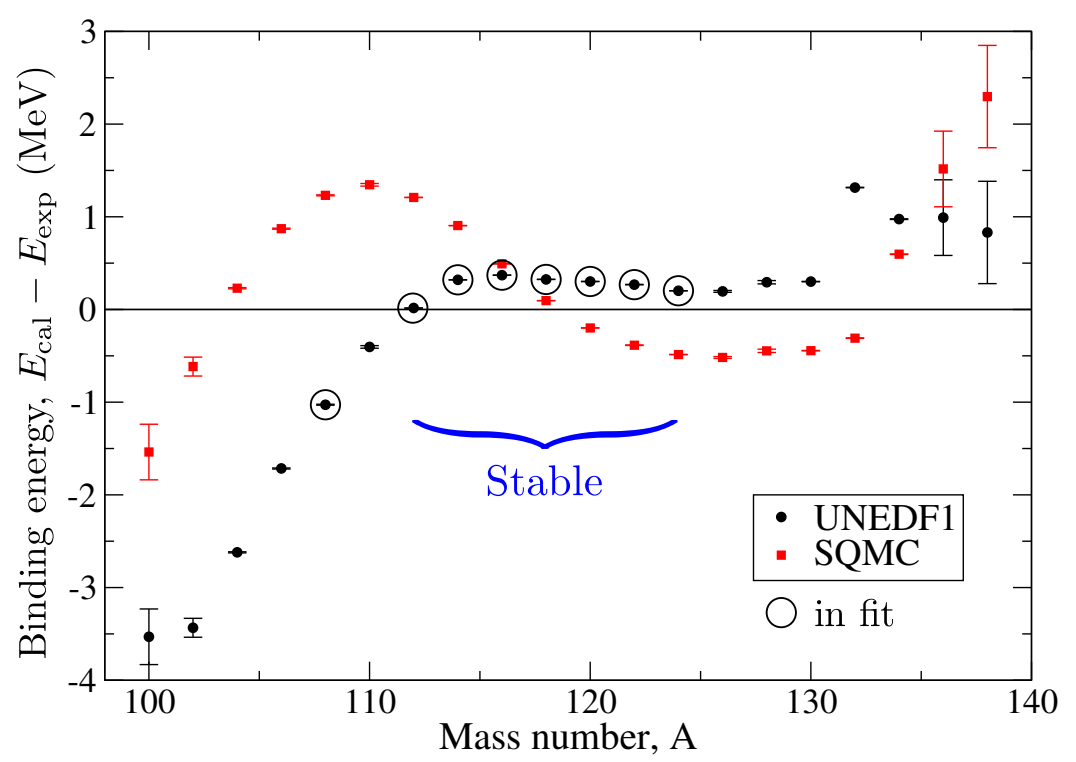

Figure 1: Difference between calculated and experimental binding energies for ${ }^{A} \mathrm{Sn}$ nuclei. The error bars are from the experimental data $\left(E_{\exp }\right)$.

It is possible to extract the impact of only the isovector dependence of the spin-orbit term by comparing a SQMC parameterisation with the isovector dependence of the QMC model $\left(W_{0}^{\prime} / W_{0}=\right.$ 1.78), against a baseline which has only one spin-orbit parameter but is otherwise identical. Calculations along the $\mathrm{Sn}$ isotopic chain out to the dripline, as shown in Figure 2, reveal that the isovector dependence of the spin-orbit term leads to a change of approximately $1 \mathrm{MeV}$ in the region of the r-process. A change half this size is expected to have a significant impact on r-process abundances [10]. This illustrates that it is crucial to properly account for the isovector properties of the spinorbit functional, as predicted by the QMC model for example. The calculations also predict that the Sn nuclei in the region from $A=146$ to 162 are noticably deformed. This deformation significantly enhances the effect of the isovector properties of the spin orbit term.

\section{Conclusions}

The isovector dependence of the spin-orbit term from the QMC model, with its high-energy degrees of freedom, is remarkably similar to that of UNEDF1. This is significant because this dependence is an important factor that should be accounted for in r-process calculations. A Skyrme 


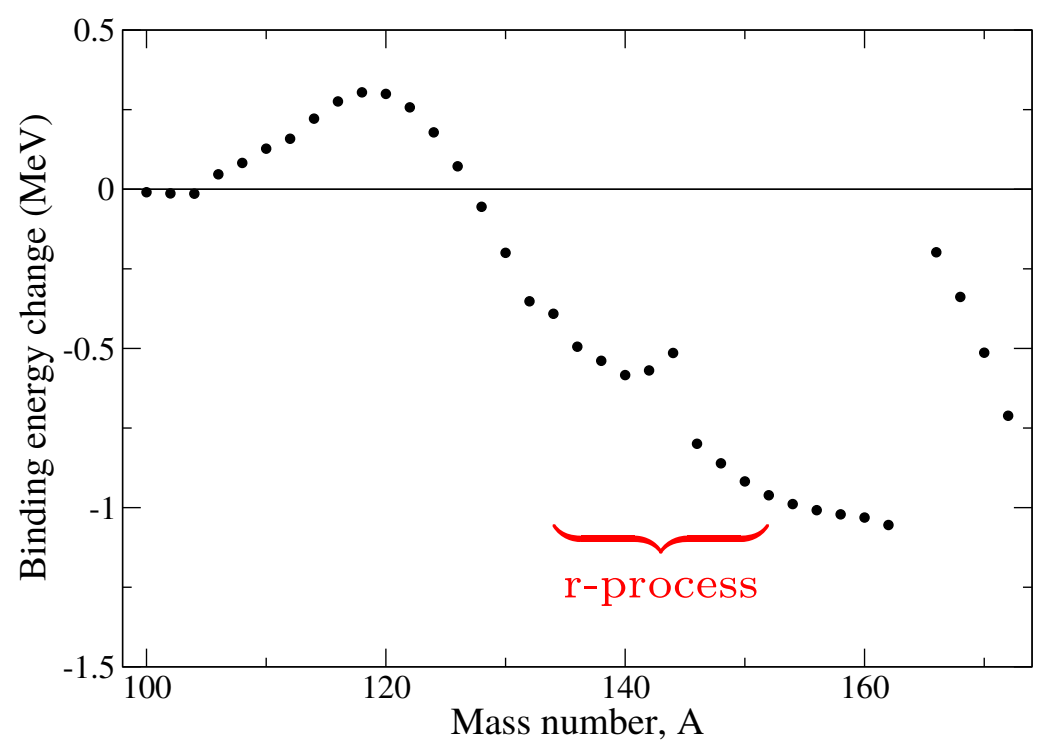

Figure 2: Binding energy difference between SQMC with $W_{0}^{\prime} / W_{0}=1.78$ and SQMC with $W_{0}^{\prime} / W_{0}=1$, for ${ }^{A}$ Sn nuclei.

functional from the QMC model can successfully reproduce ground-state binding energies. In the future, the SQMC functional will be used to study further exotic structure effects or, using a timedependent Hartree-Fock code, dynamic processes including reactions with exotic nuclei, fusion barriers, transfer and fission, revealing how they are affected by the high-energy physics the QMC model is based on. It will also be possible to perform similar investigations using the original QMC functional, rather than the SQMC parameterisation, by modifying Skyrme-based HartreeFock codes to accept the more complicated density dependence of the central terms.

\section{Acknowledgments}

This research is supported by an Australian Government Research Training Program (RTP) Scholarship and ARC Grant No. FT120100760.

\section{References}

[1] T. H. R. Skyrme, The effective nuclear potential, Nucl. Phys. 9 (1959) 615.

[2] P. A. M. Guichon, A possible quark mechanism for the saturation of nuclear matter, Phys. Lett. B 200 (1988) 235.

[3] P. A. M. Guichon, H. H. Matevosyan, N. Sandulescu, and A. W. Thomas, Physical origin of density dependent forces of Skyrme type within the quark meson coupling model, Nucl. Phys. A 772 (2006) 1.

[4] P.-G. Reinhard and H. Flocard, Nuclear effective forces and isotope shifts, Nucl. Phys. A 584 (1995) 467.

[5] M. Kortelainen, J. McDonnell, W. Nazarewicz, P.-G. Reinhard, J. Sarich, N. Schunck, M. V. Stoitsov, and S. Wild, Nuclear energy density optimization: Large deformations, Phys. Rev. C 85 (2012) 024304. 
[6] M. M. Sharma, G. Lalazissis, J. König, and P. Ring, Isospin dependence of the spin-orbit force and effective nuclear potentials, Phys. Rev. Lett. 74 (1995) 3744.

[7] X. B. Wang, C. Qi, and F. R. Xu, Isovector channel of quark-meson coupling model and its effect on symmetry energy, Nucl. Phys. A 865 (2011) 57.

[8] M. V. Stoitsov, N. Schunck, M. Kortelainen, N. Michel, H. Nam, E. Olsen, J. Sarich, and S. Wild, Axially deformed solution of the Skyrme-Hartree-Fock-Bogoliubov equations using the transformed harmonic oscillator basis (II), Comp. Phys. Comm. 184 (2013) 1592.

[9] M. Wang, G. Audi, A. H. Wapstra, F. G. Kondev, M. MacCormick, X. Xu, and B. Pfeiffer, The AME2012 atomic mass evaluation, Chin. Phys. C 36 (2012) 1603.

[10] M. R. Mumpower, R. Surman, G. C. McLaughlin, and A. Aprahamian, The impact of individual nuclear properties on r-process nucleosynthesis, Prog. Part. Nucl. Phys. 86 (2016) 86. 